\title{
ÉTUDE DE L'INFLUENGE DE LA D-L THYROXINE SUR LE POIDS DE L'OEUF, SUR LE POIDS DU JAUNE ET SA COMPOSITION.
}

\author{
Marie-Jeanne THUILLII, L. LACASSAGNE et C. CALET. \\ Aver la collaboration technique de Monique Auvray et Marie-France Lorzeat. \\ Station de Recherches avicoles, \\ Centre national de Recherches zootechniques, Jouy-en-Josas.
}

\section{SOMMAIRE}

La d-l thyroxine ingérée par des poules en ponte diminue le nombre et le poids des oufs pondus ainsi que le poids des jaunes. Malgré cette diminution, les teneurs en matière sèche, protides, lipides et acides gras des réserves vitellines demeurent constantes en général. Il y a toutefois lieu de signaler que 3 ovules sur 93 ont fait exception à cette règle.

Depuis TERROINE et BEILIN I927, on sait que l'œuf présente une constance de composition remarquable. De plus ces auteurs ont montré que l'influence de l'alimentation ne modifie en rien la composition du jaune en constituants fondamentaux qu'il s'agisse de la teneur en eau, en acides gras totaux et en cholestérol.

Nous savons d'autre part depuis les travaux de Asmundson I93 I et Asmundson et Pinsky I935, que l'ingestion de poudre de thyrö̈de séchée provoque chez la poule une diminution du poids du jaune. L'un d'entre nous a montré que l'administration de thyroxine produit un effet comparable. Sous l'action de cette hormone la réduction des réserves vitellines peut être très importante et se traduire par un abaissement de $27 \mathrm{p}$. Ioo de la matière sèche du jaune par rapport à sa valeur avant traitement hormonal (LACASSAGNE, I957). Cette diminution provient non d'une ovulation prématurée mais d'une réduction du dépôt quotidien de vitellus dans l'ovocyte (Asmundson et Pinsky, i935; LaCAssagne, I957).

On est en droit de se demander devant un abaissement aussi considérable de la matière sèche si la fixité de la composition du jaune est maintenue. Nous avons donc entrepris l'étude de cette composition chez un certain nombre de poules avant et après traitement à la thyroxine. 


\section{MATÉRIEL ETT MÉTHODES}

\section{ANIMAUX}

Dans un troupeau de pondeuses, nous avons choisi i 2 poules dont les séries étaient de 3 ou 4 œufs. Ces poules, élevées en batteries, furent partagées en deux lots de 6 animaux chacun : un lot témoin comprenant les poules $n^{\circ} 2,4,6,54,67,78$ et un lot traité comprenant les poules $n^{\circ} 9,22$, $51,61,63$ et 69 .

Les animaux étaient âgés de 2 mois au 3 I mars 1959, date du début de l'expérience; ils furent nourris ad libitum avec un régime complet équilibré dosant i 6 p. Ioo de protéines et I 800 calories productives au kg, jusqu'à la fin de l'expérience, le 7 juillet 1959 .

\section{TRAITEMENT}

Aprés une période de référence de 5 semaines, le traitement fut appliqué aux 6 poules du lot traité. La d-1 thyroxine, sous forme de pastilles, leur fut donnée à ingérer chaque soir à 18 h. Les doses administrées furent :

- la I $^{\mathrm{re}}$ semaine de I mg par jour

- la $2^{\mathrm{e}}$ semaine de 1,5 mg par jour

- la $3^{\mathrm{e}}$ semaine de $2 \mathrm{mg}$ par jour

- la $4^{\mathrm{e}}$ semaine de $2,5 \mathrm{mg}$ par jour

- la $5^{\mathrm{e}}$ et $6^{\mathrm{e}}$ semaine de 3 mg par jour

- la $7^{\mathrm{e}}$ et $8^{\mathrm{e}}$ semaine de $3,5 \mathrm{mg}$ par jour

J.'ingestion de 3 et $3,5 \mathrm{mg}$ provoquant un ralentissement net de l'ovogénèse nous avons en effet administré chacune de ces doses durant i 5 jours afin d'éviter un arrêt de la ponte.

\section{MÉTHODE}

Au cours de l'expérience tous les oufs et jaunes frais des poules traitées sont pesés ainsi que ceux des poules témoins. L'intensité de ponte est relevée.

L'étude de la teneur du jaune en lipides porte sur le premier et dernier cuf des séries des poules reccvant la thyroxine, à l'exception des œufs d'une période de transition de 7 jours correspondant à la première semaine de traitement. Cette analyse est réalisée à partir des jaunes frais à l'aide de deux techniques : méthode de Kumagawa et méthode d'extraction à froid mise au point par ThurLLIE ( 1960$)$.

Méthode de Kumagnwa. - Cette méthode donne avec la teneur en lipides et substances liposolubles, celle de la matière sèche et par déduction celle de l'eau. Elle fut appliquée aux premiers et derniers cufs, d'une série sur deux, des poules $n^{\circ} 22,5^{\text {I }}$ et 6I. Nous avons en plus effectué le dosage d'azote sur l'extrait alcoolique et le résidu délipidé par la méthode de KjeLDAHL.

Méthode d'extraction à froid. - I.e mélange méthanol, chloroforme, eaul dans les proportions 2/4/8 (vol/vol) permet d'extraire à froid les lipides totaux et substances liposolubles du jaune de l'œuf frais, avec une récupération d'environ $98 \mathrm{p}$. I oo. Nous avons utilisé cette méthode pour obtenir, après saponification, des acides gras dont la structure puisse être considérée comme intacte. Sur ces acides gras, nous avons procédé à quelques indices d'iode (adaptation de la méthode de KALFMAN), afin de compléter notre étude sur une variation éventuelle de la composition globale du jaune.

\section{RÉSULTATS}

Les variations pondérales des œufs et de leurs jaunes sont reportées, par poule, aux tableaux $x$ et 2 pour les animaux traités à la thyroxine.

Nous voyons que cette hormone provoque une diminution de la taille de l'œuf ainsi que celle du jaune. Tous les animaux ont répondu de façon homogène et la comparaison des résultats entre la période de référence et la période de traitement fait apparaître une différence significative pour chaque poule, ce qui confirme les travaux antérieurs effectués sur ce sujet. Cependant les animaux ne se trouvant pas en salles 
TABLEAU I

Poules traitées - Variation du poids moyen de l'auf, par poule, sous l'action de la thyroxine.

\begin{tabular}{|c|c|c|c|c|c|c|}
\hline \multirow{2}{*}{$\begin{array}{c}\text { Numéro } \\
\text { des } \\
\text { poules }\end{array}$} & \multicolumn{2}{|c|}{ Période de référence } & \multicolumn{2}{|c|}{ Période de traitement } & \multirow[b]{2}{*}{ Différence } & \multirow[b]{2}{*}{ Signification } \\
\hline & $\begin{array}{l}\text { Nombre } \\
\text { d'œufs }\end{array}$ & $\begin{array}{l}\text { Poids moyen des } \\
\text { ocufs en } g(\mathbf{l})\end{array}$ & $\begin{array}{l}\text { Nombre } \\
\text { d'oufs }\end{array}$ & $\begin{array}{l}\text { Poids moyen des } \\
\text { œufs en } g(1)\end{array}$ & & \\
\hline 9 & 16 & $60,11 \pm 0,33$ & 29 & $56,66 \pm 0,33$ & $-3,45$ & $\mathrm{P}<0,0005$ \\
\hline 22 & 15 & $66,15 \pm 0,45$ & 10 & $61,09 \pm 0,46$ & $-5,06$ & $\mathrm{P}<0,0005$ \\
\hline 51 & 25 & $60,79 \pm 0,29$ & 13 & $56,71 \pm 0,74$ & $-4,08$ & $\mathrm{P}<0,0005$ \\
\hline 61 & 27 & $68,6^{\prime} \div 0,30$ & 11 & $65,43 \pm 0,85$ & $-3,21$ & $\mathrm{P}<0,0005$ \\
\hline 63 & 22 & $63,23 \pm 0,36$ & 23 & $58,51 \div 0,43$ & $-4,72$ & $\mathrm{P}<0,0005$ \\
\hline 69 & 22 & $65,19 \pm 0,27$ & 5 & $59,61 \pm 1,50$ & $-5,58$ & $\mathrm{P}<0,0005$ \\
\hline
\end{tabular}

(1) Erreur égale à $\pm \frac{\sigma}{\sqrt{n}}$ dans tous les tableaux.

TABLEAU 2

Poules traitées - Variation du poids moyen du jaune frais, par poule, après ingestion de thyroxine

\begin{tabular}{|c|c|c|c|c|c|c|}
\hline \multirow{2}{*}{$\begin{array}{l}\text { Numéro } \\
\text { des } \\
\text { poules }\end{array}$} & \multicolumn{2}{|c|}{ Période de référence } & \multicolumn{2}{|c|}{ Période de traitement } & \multirow[b]{2}{*}{ Différence } & \multirow[b]{2}{*}{ Signification } \\
\hline & $\begin{array}{l}\text { Fombre } \\
\text { d'oufs }\end{array}$ & $\begin{array}{l}\text { Poids moyen des } \\
\text { jaunes ell g }\end{array}$ & $\begin{array}{l}\text { Nombre } \\
\text { d'œufs }\end{array}$ & $\begin{array}{l}\text { Poids moven des } \\
\text { jaunes en } \mathrm{g}\end{array}$ & & \\
\hline 9 & 17 & $18,44 \pm 0,92$ & 30 & $15,96 \leq 0,23$ & $-2,48$ & $\mathrm{P}<0,001$ \\
\hline 22 & 16 & $20,06 \pm 0,32$ & 10 & 17,42 는 0,37 & - 2,61 & $\mathrm{P}<0,0005$ \\
\hline 51 & 24 & $18,68 \pm 0,29$ & $l^{\prime} t$ & $15,83 \pm 0,20$ & $-2,85$ & $P<0,0005$ \\
\hline 61 & 27 & $19,25 \frac{1}{1} 0,16$ & 11 & $17,82 \pm 0,31$ & $-1,43$ & $P<0,0005$ \\
\hline 63 & 22 & $20,02 \pm 0,20$ & 23 & $17,68 \pm 0,26$ & $-2,34$ & $\mathrm{P}<0,0005$ \\
\hline 69 & 22 & $19,42 \pm 0,12$ & $\tilde{j}$ & $17,52 \pm 0,05$ & $-1,90$ & $P<0,0005$ \\
\hline
\end{tabular}

conditionnées nous avons jugé utile d'effectuer simultanément des mesures identiques sur un lot témoin. Les chiffres correspondant figurent aux tableaux 3 et 4 . Nous y remarquons que durant l'expérience l'évolution normale du poids de l'œuf et du jaune, bien qu'irrégulière selon les animaux, s'est effectuée vers une augmentation le plus souvent significative, ce qui vient renforcer les conclusions précédentes.

TABLEAU 3

Poules témoins - Évolution du poids moyen de l'œuf, par poule, durant l'expérience.

\begin{tabular}{|c|c|c|c|c|c|c|}
\hline \multirow{2}{*}{$\begin{array}{l}\text { Numéro } \\
\text { des } \\
\text { poules }\end{array}$} & \multicolumn{2}{|c|}{ Période de référence } & \multicolumn{2}{|c|}{ Période de traitement } & \multirow{2}{*}{ Différence } & \multirow{2}{*}{ Signification } \\
\hline & $\begin{array}{l}\text { Nombre } \\
\text { d'œufs }\end{array}$ & $\begin{array}{l}\text { Poids moyen des } \\
\text { ceufs en } g\end{array}$ & $\begin{array}{l}\text { Nombre } \\
\text { d'œufs }\end{array}$ & $\begin{array}{l}\text { Poids moyen des } \\
\text { œulf en } g\end{array}$ & & \\
\hline 2 & 17 & $64,87 \pm 0,12$ & 25 & $65,41 \pm 0,89$ & $+0,57$ & NS \\
\hline 4 & 13 & $59,38 \pm 0,71$ & 32 & $61,41+0,18$ & $\begin{array}{r}+2,03 \\
+\quad 2\end{array}$ & $\mathrm{P}<0,005$ \\
\hline 54 & 19 & $59,99 \pm 0,31$ & 29 & $59,73 \pm 0,45$ & $-0,26$ & $\mathrm{NS}$ \\
\hline 67 & 19 & $64,41 \pm 0,59$ & 25 & $65,91 \pm 0,49$ & $+1,53$ & $\mathrm{P}^{3}<0,025$ \\
\hline 78 & 16 & $64^{\prime}, 28 \pm 0, y^{\prime}+4$ & 31 & $67,04 \pm 0,81$ & $+2,76$ & $\mathrm{P}<0,005$ \\
\hline
\end{tabular}


TABLEAU 4

Poules témoins — Évolution du poids moyen des jaunes frais, par poule, durant l'expérience.

\begin{tabular}{|c|c|c|c|c|c|c|}
\hline \multirow{2}{*}{$\begin{array}{c}\text { Numéro } \\
\text { des } \\
\text { poules }\end{array}$} & \multicolumn{2}{|c|}{ Période de référence } & \multicolumn{2}{|c|}{ Période de traitement } & \multirow{2}{*}{ Différence } & \multirow{2}{*}{ Signification } \\
\hline & $\begin{array}{l}\text { Nombre } \\
\text { d'cufs }\end{array}$ & $\begin{array}{l}\text { Poids moyen des } \\
\text { jaunes en } g\end{array}$ & $\begin{array}{l}\text { Nombre } \\
\text { d'œufs }\end{array}$ & $\begin{array}{l}\text { Poids moyen des } \\
\text { jaunes en } g\end{array}$ & & \\
\hline 2 & 17 & $20,95 \pm 0,23$ & 25 & $20,71 \pm 0,25$ & $-0,24$ & $\mathrm{P}<0,025$ \\
\hline 4 & 13 & $18, \mathbf{k}^{\prime} \pm 0,18$ & 32 & $19,06 \pm 0,12$ & $+0,62$ & $\mathrm{P}<0,005$ \\
\hline 54 & 19 & $19,36 \pm 0,27$ & 29 & $19,66 \pm 0,31$ & $+0,30$ & NS \\
\hline 67 & 19 & $20,10 \pm 0,26$ & 25 & $20,91 /=0,29$ & $+0,84$ & $\mathrm{P}<0,025$ \\
\hline 78 & 16 & $19,78+0,26$ & 31 & $20,26 \pm 0,19$ & $+0,48$ & NS \\
\hline
\end{tabular}

Les résultats de l'analyse du jaune de l'œuf par les deux méthodes sont rapportés dans les tableaux 5 et 6 . Dans le premier figurent les quantités et teneurs en matière sèche, lipides, protides des réserves vitellines des oufs pondus par les poules $\mathrm{n}^{0} 22$, 5 I et 6 I durant la période de référence et après ingestion de thyroxine.

TABLEAU 5

Influence de la thyroxine sur la composition du jaune de l'œuf.

\begin{tabular}{|c|c|c|c|}
\hline & Poule no 22 & Poule $n^{0} 51$ & Poule $n^{0} 61$ \\
\hline \multicolumn{4}{|l|}{ Poids du jaune frais (g) } \\
\hline Période de référence & 19,484 & 18,120 & 18,902 \\
\hline Période de traitement $\ldots \ldots \ldots \ldots \ldots \ldots \ldots \ldots \ldots$ & 17,164 & 15,660 & 17,942 \\
\hline \multicolumn{4}{|l|}{ Quantité de matière sèche (g) } \\
\hline Période de référence $\ldots \ldots \ldots \ldots \ldots \ldots \ldots \ldots \ldots \ldots$ & 10,311 & 8,898 & 9,628 \\
\hline Période de traitement $\ldots \ldots \ldots \ldots \ldots \ldots \ldots \ldots \ldots \ldots$ & 8,974 & 7,490 & 9,007 \\
\hline \multicolumn{4}{|l|}{ Teneur en matière sèche (p. 100) } \\
\hline Période de référence $\ldots \ldots \ldots \ldots \ldots \ldots \ldots \ldots \ldots \ldots \ldots \ldots \ldots \ldots \ldots \ldots$ & 52,920 & 49,215 & 50,900 \\
\hline Période de traitement $\ldots \ldots \ldots \ldots \ldots \ldots \ldots \ldots$ & 52,250 & 47,860 & 50,240 \\
\hline \multicolumn{4}{|l|}{ Quanitié de lipides totaux (g) } \\
\hline Période de référence ...... & 7,515 & 6,627 & 7,070 \\
\hline Période de traitement $\ldots \ldots \ldots \ldots \ldots \ldots \ldots \ldots \ldots$ & 6,640 & 5,500 & 6,647 \\
\hline \multicolumn{4}{|l|}{ Teneur en lipides totaux (p. 100) } \\
\hline Période de référence $\ldots \ldots \ldots \ldots \ldots \ldots \ldots \ldots \ldots$ & 38,570 & 36,700 & 37,350 \\
\hline Période de traitement $\ldots \ldots \ldots \ldots \ldots \ldots \ldots \ldots \ldots$ & 37,900 & 35,110 & 37,120 \\
\hline \multicolumn{4}{|l|}{ Quantité de protides (g) } \\
\hline Période de référence $\ldots \ldots \ldots \ldots \ldots \ldots \ldots \ldots \ldots$ & 2,768 & 2,197 & 2,400 \\
\hline Période de traitement $\ldots \ldots \ldots \ldots \ldots \ldots \ldots \ldots$ & 2,227 & 1,970 & 2,283 \\
\hline \multicolumn{4}{|l|}{ Teneur en protides (p. 100) } \\
\hline Période de référence $\ldots \ldots \ldots \ldots \ldots \ldots \ldots \ldots \ldots$ & 14,206 & 12,125 & 12,685 \\
\hline Période de traitement $\ldots \ldots \ldots \ldots \ldots \ldots \ldots \ldots \ldots$ & 12,750 & 12,570 & 12,820 \\
\hline
\end{tabular}

Sous l'effet du traitement nous assistons à la fois à une diminution du poids du. jaune frais et de son extrait sec. Nous constatons de même une diminution des lipides totaux et des matières azotées totales. Cette réduction porte de façon égale sur tous les constituants du jaune : on observe en effet que les teneurs en matière sèche, en lipides et protides ne sont pas significativement différentes avant et après ingestion de thyroxine. Ce résultat est peut-être dû au faible nombre d'échantillons ; c'est la 
raison pour laquelle les écarts types des moyennes n'ont pas été portés au tableau. Toutefois les différences demeurent peu importantes. Il y a lieu de remarquer la grande variabilité des résultats en ce qui concerne les protides puisque selon les poules les variations d'une période à l'autre sont positives ou négatives et peuvent même atteindre ro p. roo de la valeur initiale (cas de la poule $n^{\circ} 22$ ).

Dans les échantillons analysés par la méthode à froid les dosages ne portent que sur la teneur en lipides.

\section{TABLEAU 6}

\begin{tabular}{|c|c|c|c|c|c|c|}
\hline \multirow{2}{*}{$\begin{array}{l}\text { Numéro } \\
\text { des poules }\end{array}$} & \multicolumn{2}{|c|}{ Période de référence } & \multicolumn{2}{|c|}{ Période de traitement } & \multirow[b]{2}{*}{ Différence } & \multirow[b]{2}{*}{ Signification } \\
\hline & $\begin{array}{l}\text { Nombre de } \\
\text { jaunes }\end{array}$ & Lipides en p. 100 & $\begin{array}{c}\text { Nombre de } \\
\text { jaunes }\end{array}$ & Lipides en $\% 100$ & & \\
\hline 9 & 14 & $35,24=0,28$ & 28 & $33,40 \perp 0,29$ & $-1,34$ & $\mathrm{P}^{\prime}<0,001$ \\
\hline 22 & 7 & $33,80 \perp 0,70$ & 6 & $35,11 \div 0,69$ & $+1,31$ & $\mathrm{NS}$ \\
\hline 5 & 8 & $35,05=0,60$ & 8 & $3 x^{\prime} f_{i} \pm 0,95$ & $-0,59$ & $\times S$ \\
\hline 61 & 7 & $34,56 \div 0,75$ & 5 & $34,93+0,22$ & $+0,37$ & $\therefore \mathrm{s}$ \\
\hline 63 & $1 t^{\prime}$ & $34,47+0,49$ & 19 & 35,10 - $1,0,23$ & $+0,63$ & $\mathrm{NS}$ \\
\hline 69 & 16 & $35,12 \pm 0,32$ & 5 & $3,12 \perp 2,10$ & $-1,00$ & sis \\
\hline
\end{tabular}

Les données établies par cette méthode appellent quelques remarques. En valeurs absolues, les teneurs enregistrées sont toujours plus faibles que celles obtenues par la méthode classique de Kumagawa. Ceci provient, en partie, de ce que le pourcentage de récupération des lipides, à froid, ne dépasse pas 98 p. Ioo. Dans tous les cas sauf un, celui de la poule $n^{\circ} 9$, nous n'avons pu établir de différences significatives avant et après traitement, les variations sont positives ou négatives selon l'anima1 considéré.

Seuls deux animaux ont continué à pondre longtemps sous l'action des doses croissantes de thyroxine. Il s'agit des poules no 9 et 63 dont les réponses, en ce qui concerne la teneur en lipides, sont diamétralement opposées.

Les poules no 9 et 69 ont pondu, l'une deux cufs à 29,7 p. roo de lipides, l'autre un œuf à 26,6 p. Ioo après un arrêt de cinq jours. Les différences enregistrées pour ces deux animaux entre les deux périodes d'expérience proviennent essentiellement de ces trois données.

En ce qui concerne les teneurs en acides gras, les chiffres sont respectivement, avant et après traitement à la thyroxine, de $82,79 \pm 0,32$ et $83,12 \pm 0,59$. L'égalité des deux moyennes et 1 'homogénéité des résultats nous amènent à une double conclusion :

- il n'y a pratiquement aucune différence de cette teneur entre animaux ;

- la teneur de ces acides demeure inchangée sous l'action de la thyroxine.

La détermination de l'indice d'iode durant ces deux périodes, n'a pu être effectué sur tous les jaunes. Malgré le peu de résultats obtenus, cet indice, pour un régime donné, semble constant chez une même poule, mais varie d'un animal à l'autre, ce qui est en accord avec les résultats de MotrRAM, IgI3. 


\section{DISCUSSION}

Les résultats se rapportant à la diminution de poids des œufs et du jaune confirment ceux déjà obtenus par de nombreux auteurs, bien que chaque animal ait eu un comportement différent sous l'effet de la thy roxine. En effet, si l'on considère la durée de la période de ponte. la poule no 69 a pondu I2 oeufs dans les 33 premiers jours du traitement puis s'est arrêtée, alors que la poule $n^{0} 9$ a produit 37 œufs en 6 I jours. I a ponte des autres animaux se situe entre ces deux limites.

Le fait remarquable est la constance de composition des réserves vitellines, qui ressort de l'ensemble des tableaux 5 et 6 , malgré l'abaissement très sensible du poids du jaune et un arrêt souvent rapide de la ponte. Ceci est vraisemblablement dû̀ à ce que la thyroxine n'a aucune action sur l'épithélium folliculaire. L'hormone agirait essentiellement par diminution de la quantité d'aliment ingéré (Asmundson et PINSKY, I935 ; LACASSAGNE, non publié), par augmentation du catabolisme et inhibition plus ou moins complète des modifications sanguines qui accompagnent la ponte (Hosoda et al., I954) ou qui suivent l'administration d'œstrogènes (FLEISCHMANN et FrIED, I945 ; Mc DONALd, RIDDLE et Smith, I945 ; Hosoda et a1., I954).

I1 semble cependant que par exception, la teneur des réserves vitellines puisse varier. C'est le cas des poules $n^{\circ} g$ et 69 que nous avons déjà citées. Elles ont pondu, l'une deux oufs dont les jaunes renfermaient 29,7 p. Ioo de lipides et l'autre un œuf dont la jaune n'en contenait que 26,6 p. Ioo. Ce dernier ovule a vraisemblablement eu une évolution anormale. Il précédait en effet l'arrêt définitif de ponte et a été pondu cinq jours après l'avant dernier ouf. Il n'en est pas de même des deux ovules de la poule $n^{\circ} 9$ qui malgré leur teneur lipidique très basse provenaient de séries normales, cet animal ayant continué à pondre durant toute la période de traitement à la thyroxine.

$$
\text { Reçu en février } 1960
$$

\section{SUMMARY}

A STUDY OF THE EFFECT D-L. THYROXIN FED TO LAYING-HENS ON EGG-WEIGIIT, YOLK-WEIGHT AND THE COMPOSITION OF YOLK

Six one year old Rhode $\mathrm{x}$ Wyandotte laying hens were fed increasing amounts of $\mathbf{d}$-l thyroxine (from $\mathrm{r}$ to $3,5 \mathrm{mg}$ per day) during 8 weeks.

When this hormone is fed, a decrease in weight and egg production is observed, together with a decrease in the weight of the yolk and its components.

On the other hand, no significant variation in the dry-matter, protein, lipid, and total fattyacid percentages of the ovul could be evidenced.

The specificity of individual birds'responses is discussed.

\section{RÉFÉRENCES BIBLIOGRAPHIQUES}

Asmundon V.S., I93I. Effect of hormones on the formation of the hen's egg. Poult. Sci., 10, i57-I65. AsMundson V.S., Pinsky P., I935. 'The effect of the thyroid on the formation of the hen's egg. Poult. Sci., 14, 99-IO4.

FleischmanN W., Fried I. A., 1945. Studies on the mechanism of the hypercholesterolemia and hypercalcemia induced by estrogen in immature chicks. Endocrinology, 36, 405-4I 5. 
Hosoda T., Kakneo T., Magi K., ABE T., I954. Effect of thyroxine on vitellin production in the fowl. Section papers tenth World's poultry congress, $\mathrm{p}$. $\mathrm{1} 34-\mathrm{r} 36$.

LACASSAGNE L., 1957. Action de la $d$-l thyroxine sur la durée d'évolution de la phase de grand accroissement du follicule chez la poule domestique. C.R. Acad. Sci., 45, I830-1832.

MCDONALD M. R., RIDDLE O., SMITII G. C., 1945. Action of thyroxin on oestrogen-induced changes in blood chemistry and endosteal bone. Endocrinology, 37, 23-28.

MotTRAM V. A., I9r3. Fatty acids of hen's eggs. J. P.hysiol. (G. B.), 47, XVIII-XIX.

TERROINE E. F., BEI.IN P., rg27. Influence de l'alimentation sur la composition quantitative de l'œuf de poule. Bull. Soc. Chim. Biol., 9, 1074-1084. 